\title{
La utilización del modelo de gobernanza policéntrica, de Elinor Ostrom, como medio de salvaguardar la efectividad de la gestión democrática de las cooperativas en las situaciones de representación delegada
}

\author{
Leonardo Rafael de Souza' \\ Alumno del programa de maestría en Gestión de Cooperativas \\ de la Pontifícia Universidade Católica do Paraná - PUC/PR
}

Sumario: 1. Introducción. 2. De la Teoría de los Recursos Comunes a la propuesta de un modelo de gobernanza policéntrica para la gestión de las propiedades colectivas. 3. La Gestión Democrática como premisa de las sociedades cooperativas. 4. La sociedad cooperativa en cuanto bien común y la policentricidad como fuente de defensa de la gestión democrática. Reflexión estimulada por Elinor Ostrom. 5. Consideraciones y reflexiones finales. 6. Referencias.

Resumen: De acuerdo con el principio de gestión democrática, las cooperativas son organizaciones gestionadas por sus miembros, los cuales participan activa y directamente en la determinación de sus políticas y en la toma de decisiones. Sin embargo, cuando la sociedad cooperativa asciende considerablemente, en términos de cobertura territorial o de cantidad de miembros, la Ley de Cooperativas brasileña establece la posibilidad de la representación delegada en el ámbito de las asambleas generales. En este sentido, el presente trabajo tiene como objetivo analizar el modelo de gobernanza policéntrica, de Elinor Ostrom, utilizándole como palanca de sustentación de la gestión democrática de las sociedades cooperativas consideradas de grande porte, que adoptan la posibilidad de representación delegada. El objetivo es confirmar que la adopción de la representación delegada conforma un sistema de gobernabilidad pluridemocrática y sistémica que tanto no afecta la esencia participativa del conjunto de miembros, como resguarda la democracia de la gestión preconizada por la máxima de un hombre un voto. Para materializar la investigación se va a utilizar el método inductivo de abordaje, con empleo de la investigación directa e indirecta.

1 E-mail: leonardorafae/@sdl.adv.br 
Palabras clave: Cooperativismo. Gestión Democrática. Representación Delegada. Gobernanza Policéntrica. Elinor Ostrom.

Abstract: In accordance with the principle of democratic management, cooperatives are organizations controlled by their members, who participate actively and directly in determining their policies and in making decisions. However, when the cooperative society increases considerably in terms of territorial coverage or number of members, the law of Brazilian cooperatives establishes the possibility of delegated representation for the holding of general assemblies. In this sense, this article aims to analyze the model of polycentric governance developed by Elinor Ostrom, using it as a theoretical alternative to explain the democratic management of large cooperative societies that have the opportunity to delegate representation. The aim is to confirm that the adoption of delegated representation forms a multidemocratic governance system that not only does not affect the participatory essence of all members but also protects democracy in cooperative management advocated by the maxim of one man one vote. To materialize the research, we will use the inductive approach method with indirect research use.

Keywords: Cooperativism. Democratic management. Delegated Representation. Polycentric Governance. Elinor Ostrom. 


\section{Introducción}

Las sociedades cooperativas alrededor del mundo a partir de su concepción moderna construida desde la Sociedad Equitativa de los Pioneros de Rochdale ha evolucionado de forma significativa en su representatividad económica y social. Están consolidadadas en la defensa colectiva de la fuerza humana y en un modelo económico hecho por y para el hombre, promoviendo la universalización del acceso a los mercados (MIRANDA, 2012). Como consecuencia de eso se observa en los números del Cooperativismo mundial.

Según el Censo Global de las Cooperativas realizado en 2014 por el Secretariado General de las Naciones Unidas, hay en el mundo más de 2,6 millones de cooperativas, las cuales congregan aproximadamente mil millones de miembros y clientes y, aún permiten el acceso al trabajo por más de 12,6 millones de personas. Bajo el punto de vista económico, esas cooperativas han generado alrededor de US\$2,98 billones de ingreso anual, decorrentes de activos combinados de US\$19,6 billones, números mayores que la economía de Francia. Aún de acuerdo con la entidad, a pesar de que las cooperativas sean en su gran mayoría empresas de propiedad local y gobernada por sus propios miembros, en paises como Francia y Finlandia los emprendimientos cooperativos representan un $10 \%$ más del Producto Interno Bruto (PIB). Solo en Nueva Zelanda este porcentaje llega a un 20\% (ONU, 2014).

Considerando la división de las cooperativas en sectores de actuación reconocidos por la Alianza Cooperativa Internacional $(\mathrm{ACl})$, las cooperativas agropecuarias y financieras son las que más se destacan, respectivamente, en números de cooperativas (más de 1 millón de cooperativas agropecuarias) y cantidad de miembros (más de 700 millones de asociados en cooperativas financieras) (ONU, 2014). En Brasil, esta tendencia se repite y de las 6.655 cooperativas existentes, 1.555 (alrededor de un $23 \%$ ) son cooperativas agropecuarias, mientras que de los 13,2 millones de cooperados actualmente vinculados a alguna cooperativa, un 56,5\% (7.476.308 miembros) están distribuidos en las 976 cooperativas de crédito existentes (OCB, 2016).

Del mismo modo, dados actuales presentados por la ICA (2016) muestran que de los valores mencionados y actualizados por el censo de la ONU, gran parte de las facturaciones de esas cooperativas ocurre en grandes estructuras, entendidas no solamente por sus activos, como también por el número de asociados. El referido documento revela que solo las 300 mayores cooperativas del mundo presentaron en 2016 una facturación de US $\$ 2,53$ mil millones, con un crecimiento anual superior a un 7\% (ACl, 2016). 
Sin embargo, este crecimiento económico provoca una necesaria reflexión sobre cómo las grandes cooperativas garantizan no solamente el pleno ejercicio de su papel social, pero también y principalmente la aplicabilidad del principio de la gestión democrática en la definición de los rumbos de la empresa común. Eso porque es deber de toda empresa cooperativa fomentar la efectiva participación de los asociados en los procesos de toma de decisiones alrededor del globo, visto que la democracia es un valor cooperativo fundamental en la medida en que posee un carácter estructural vinculado a la propia organización de la empresa cooperativa, además de ser la garantía de que esta sociedad esté a servicio de las personas (BÖÖK, 1992; MIRANDA, 2012).

Esa reflexión, a propósito, fundamenta la propia ponderación presentada por la $\mathrm{ACl}$ en su «Plan de Acción para una Década Cooperativa». En este documento, la entidad máxima del Cooperativismo mundial ha justificado que la protección de la democracia cooperativa está en el hecho de que el miembro, individualmente, desempeñe un papel que ultrapasa la simple relación económica básica de cliente, trabajador o produtor. En una cooperativa, los miembros actúan colectivamente a través de sus compromisos democráticos que garantizan a todos los miembros, información, voz y representación, independientemente del tamaño de la cooperativa (ACl, 2013).

Además, la defensa y la promoción de la participación del miembro se muestra todavía más inmediata en el mundo contemporáneo donde las distancias se acortan, la multiplicidad de proveedores en competencia y el actual poder de la internet sustituyeron de forma considerable la escassez de antaño por una amplia gama de elecciones dentro de una cultura consumista. Como consecuencia de eso se tiene la reducción de iniciativas de autoayuda en el ámbito comunitario, en un nivel de individualismo que tiene la «tendencia de volvernos apáticos, complacientes, o totalmente perezosos, desalentando la participación cívica, y estimulando la continuación del placer personal y de satisfacción» (ACl, 2013, p. 9).

Es en el combate a esta tendencia que está la justificación práctica de este estudio, es en el enfrentamiento de la apatía social perceptible en la sociedades cooperativas que se apoya el fortalecimiento de la gestión democrática. Desafío este puesto por la $\mathrm{ACl}(2013$, p. 10) en su Plan de Acción:

En este contexto, la participación -incluyendo una amplia participación democrática- se convierte un fin en sí mismo, una forma de enfrentar la acumulación de poder en las manos de una 
pequeña élite, y una vía para contestar los métodos absoletos de las generaciones precedentes que parecen haber fracasado. Capacita los individuos para tener algún nivel de influencia sobre las cosas que afectan sus vidas. Eso va al encuentro de la necesidad de participación democrática en una amplia gama de instituciones de que los individuos presentemente se sienten excluidos, y que parece ni siquiera darse cuenta de la situación. De ese modo la participación vuelve a ser uno de los más valiosos trunfos del sector cooperativo.

En lo que se refiere a la instrumentalización de la democracia a través de la efectiva participación, el derecho de decisión por el voto siempre fue la premisa del movimiento cooperativo desde las aprobaciones asambleares de Rochdale (HOLYOAKE, 1975), asegurando la $\mathrm{ACl}$ (2013) que tal expresión democrática no debe ser abandonada. Contodo, la propia entidad reconoce que el mundo moderno trae al voto nuevas formas de participación y empeño a partir de innovaciones, las cuales necesitan ser asumidas por las cooperativas, incluso como forma de inspirar e involucrar la nueva generación de miembros. Esto está en la esencia cooperativa en la medida en que la participación democrática de los miembros por el simple hecho de ser miembros es justamente la acción consciente fundamental que distingue el modelo empresarial cooperativo de las demás estructuras empresariales existentes (SCHNEIDER, 1991).

Contodo, si la democracia es un valor ontológico que conforma la ideología cooperativa y la formación de grandes sociedades cooperativas es una realidad inevitable, se vuelve necesario analisar y desafiar las prácticas existentes de democracia participativa en busca de alternativas innovadoras que bien comprendan la singularidad del modelo cooperativo para así «elevar la participación y la gobernanza de los miembros a un nuevo escalón (ACl, 2013, p. 6).

De los modelos alternativos discutidos hace años está la representación delegada, cuya matriz conceptual está en la posibilidad de la cooperativa realizar una asamblea compuesta por delegados elegidos por los propios asociados cuando el número de miembros supera el número establecido por la respectiva legislación y/o cuando estos viven en locales distantes. Ese respectivo procedimiento está a cargo de cada estatuto o reglamento (CRACOGNA; HENRY; FICI, 2013). Esta es la premissa legal en Brasil.

Según los párrafos del artículo 42 de la Ley 5.764 (BRASIL, 1971), cuando el número de miembros en las cooperativas singulares exceden a 3.000, o cuando aún en número inferior los asociados estén entre sí 
a una distancia superior a $50 \mathrm{~km}$, los cooperados pueden establecer, mediante aprobación estatutaria, que los mismos sean representados en las asambleas generales por delegados igualmente miembros, con algunas resalvas relacionadas a la incompatibilidad. Por otro lado, las reglas para aplicación de esa representación está a cargo del cuerpo social.

Ocurre que esa libertad dada por los legisladores a los propios miembros se tradujo, en la práctica, en una ausencia de definidos criterios para el ejercicio de la democracia cooperativa a través de la representación delegada. Consecuencia de eso es la perceptible privación de fundamentos teóricos pensados específicamente para traducir, en la representación delegada de grandes cooperativas, la máxima rochdaleana de "un hombre un voto». Aquí está, por lo tanto, la justificación teórica del presente artículo al paso que a partir de los anhelos de la $\mathrm{ACl}$ es necesario pensar estructuras democráticas propias que permitan a las cooperativas «que la participación individual lleve a una influencia real en la empresa a através de una autoridad democráticamente legitimada» ( $\mathrm{ACl}, 2013$, p. 8).

Así que, en la libertad de buscarse una base teórica de fundamentación del modelo de representación delegada — que respete la multicomplejidad de las grandes cooperativas sin alejar el precepto democrático que orienta el Cooperativismo- este estudio se propone a analisar si el modelo de gobernanza policéntrica desarrollado por Elinor Ostrom en su Teoría de los Recursos Comunes (Common Pool Resources) puede apoyar al movimiento cooperativo en la ampliación de la participación del miembro en los procesos de toma de decisiones de las sociedades cooperativas.

Esta elección se justifica por el apariente acercamiento de la ideología cooperativa con los fundamentos de la teoría de Ostrom, la cual siempre se basó en la necesidad de elaboración de sistemas de gestión de recursos que se propone no solo al crecimiento económico, sino también al incentivo a la reducción de la degradación ambiental y, principalmente, a la mejora del bienestar social de las comunidades humanas. Para la construcción de su teoría, Ostrom (2005) ha enumerado un conjunto de principios y de reglas de propiedad colectiva volcadas a la eliminación de la exploración, las cuales serán mejor profundizadas.

Como forma de delimitación metodológica de acercamiento de esa teoría a la realidad de la investigación, se considera que la democracia en cuanto valor cooperativo fundamental y estructural de la sociedad cooperativa, es un recurso común de esta relación a sus asociados y en el ejercicio de la intercooperación. Y esta definición se revela a través 
de los estudios de Ostrom al entender que cuando la identificación del recurso común está bien definido, acepto e respetado por la colectividad, se puede evitar un ataque a través de una gestión eficiente, eliminando gradativamente los conflictos por medio de la organización y de la cooperación entre individuos y/o instituciones que necesitan de este mismo recurso y que están dedicados a hacerlo de una forma sustentable (OSTROM, 2010).

Por lo tanto, este ensayo se propone no solo reflexionar sobre el modelo de gobernanza policéntrica desarrollada por Elinor Ostrom en la conformación de su Common Pool Resources, como también comprender la representación delegada dentro de la gestión democrática en cuanto premisa de las sociedades cooperativas y, así, analisar sus afinidades, reflexionando, al final, sobre la posibilidad de la matriz teórica de la gobernanza policéntrica poder justificar la construcción de modelos propios de gestión democrática en cuanto forma de preservación y fortalecimiento del valor cooperativo de la democracia.

Para la realización de este análisis se utilizará el método inductivo, en el cual la respuesta al problema aquí propuesto decorrerá de los análisis propuestos, conformando el acercamiento de teorías que podrán ser utilizadas empíricamente en futuros estudios de casos para saber si el modelo de gobernanza policéntrica en la gestión del bien común se asocia con la realidad y con la complejidad del emprendimiento cooperativo, especialmente en grandes cooperativas que necesiten de la representación delegada - ya autorizada por ley- para fundamentar el sagrado ejercicio del voto por los miembros, visto que, la garantía de la democracia en las cooperativas ocurre cuando cada miembro tiene una voz igual en lo que sucede, escucha y se comunica abierta y honestamente $(\mathrm{ACl}, 2013)$.

\section{De la Teoría de los Recursos Comunes a la propuesta de un modelo de gobernanza policéntrica para la gestión de las propiedades colectivas}

Cuando en 2009, por primera vez en la historia, una mujer fue galardonada con el premio Nobel de Economía, compartiéndolo con el consagrado economista estadounidense Oliver Williamson (1996), considerado el padre de la economía neo-institucionalista y el precursor del análises de costos de transación, el mundo de las ciencias económicas pasa a observar con mucho más atención como esta cientista política, mediante investigaciones empíricas multidisciplinares, se opuso con 
tanto reconocimiento a los modelos ortodoxos del homo economicus a través de la constatación de los aspectos comunitarios del comportamiento humano (LAURIOLA, 2009).

Con formación en Ciencias Políticas por la University of California, Los Angeles (UCLA) en 1954, Elinor se acercó a la administración pública tras profundizar sus estudios sobre la Gestión de Rercursos Hídricos, especialmente en 1965 cuando al comenzar su tesis doctoral, estudió empreendedorismo institucional dedicado a impedir la entrada de agua salada en una cuenca subterránea de agua potable en la región metropolitana de Los Ángeles (SALLES, 2009). Así pues, el periodo en que Elinor Ostrom estuvo en la UCLA le proporcionó algunas experiencias: el interés por la administración pública y por la economía, la continuación de los estudios sobre los problemas de acción colectiva enfrentados por individuos que usan recursos comunes, y también ha conocido Vicent Ostrom, con quien se ha casado (OSTROM, 2010).

Según Salles (2009), Elinor Ostrom se muda para Bloomington para acompañar a su esposo Vincent en el departamento de Ciencia Política de la Indiana University, y en esa ocasión analisa un recurso común no ambiental —la policía - presentando resultados que sirvieron de base para la toma de decisiones sobre la centralización de departamentos. Dentro de una confirmación empírica de sus estudios, diversos colegas vinculados a la Indiana University comenzaron a analizar casos relevantes sobre los recursos comunes, llegando a casi 5.000 casos en 1989, en diversas areas como sociología rural, antropología, historia, economía, ciencia política, ecología humana, entre otras. Consecuencia de esa rica construcción fue una increíble cantidad de conocimiento especializado acumulado, que ha culminado en la pionera obra Governing the Commons:The Evolution of Institutions for Collective Action, lanzado eu su primera edición en 1990 (OSTROM, 2010).

A partir de esta sólida construcción, Elinor se afianzó como una especie de musa intelectual de un movimiento de gran diversidad disciplinar y profesional, desarrollando trabajos, reflexiones e investigaciones que partieron de la insatisfacción con las lecturas y modelos ortodoxos sobre el uso y la gestión de recursos naturales. En este sentido, Lauriola (2009) y Ostrom (2010) aseguran que la ciencia económica dominante, de inspiración neoclásica, abordaba el tema a partir de tres preceptos fundamentales y complementares: (I) la tragedia de bienes comunes («The Tragedy of the Commons»); (ii) el dilema del prisionero en la teoría de los juegos, y (iii) el abordaje de Ronald Coase sobre los derechos de la propiedad. 
Sin embargo, como objetivo de desafiar la teoría de la tragedia de los bienes comunes de Garrett Hardin (1965)², que la construcción teórica y empírica de Elinor Ostrom, descripta brevemente, pasó a perseguir una nueva forma de gestionar recursos comunes, en comunidad y de modo sustentable, en la cual el adecuado conocimiento de los recursos y la consciencia de su escasez promovería una buena gestión de los mismos.

De acuerdo con Ostrom (2010), esa conciencia llevaría a un cambio de comportamiento de los agentes en las comunidades, que se alejarían del egoísmo y del individualismo para implementar reglas y principios basados en la cooperación, que es la esencia de la acción humana. De esa manera estaba estructurada la Teoría de los Recursos Comunes, originalmente Common Pool Resources.

Así como está en su nombre, la Theory of Common Pool Resources parte del propio análisis cuanto a la conceptualización y a la identificación del bien común a través de un estudio amplio e interdisciplinar. En este sentido, Hess y Ostrom (2007) elucidan que la noción del bien común -traducción libre para Commons - se refiere a un recurso compartido por un grupo de personas ${ }^{3}$, que puede ser: (I) natural, como los recursos hídricos, las florestas y la vida animal, y/o (ii) inmateriales/sin límites claros, como la capa de ozono y el conocimiento. Del mismo modo, dicen las autoras, también la amplitud de este bien común puede variar entre aquellos servibles a un pequeño grupo, a una comunidad o hasta mismo en niveles internacionales y mundiales, como son los mares profundos, la atmósfera y la internet.

Independiente de la clasificación o la amplitud de esos bienes comunes en la construcción y confirmación de la teoría, se ha comprendido que ciertos comportamientos humanos individualistas llevan a la colectividad a dilemas sociales como la competencia por el

2 Según Lauriola (2009), la Teoría afirma que frente a un recurso común cada indivíduo sigue racionalmente una lógica del beneficio individual de externalización de los costos e internalización de los beneficios, el cual practicado colectivamente conduce a la tragedia del agotamiento de este recurso. Como posibles soluciones para evitar esta tragedia, Hardin defendía una gestión racional a partir de dos premisas: (i) la del socialismo mediante intervención directa del Estado, o a de la (ii) privatización, mediante gestión descentralizada por el propio mercado.

3 Hess y Ostrom (2007) también aseguran que independientemente de su clasificación, los análisis sobre los bienes comunes casi siempre se preocupan en identificar si esos bienes son de acceso común, o sea, accesibles altivamente de su propiedad, o si son de propiedad común, las cuales exigirán reflexión anterior sobre el régimen legal de ese derecho de propiedad. 
uso y el comportamiento oportunista (free riding), causando graves impactos sobre los bienes comunes de acuerdo con su naturaleza, como por ejemplo, la mercantilización, la degradación, la polución y la insustentabilidad. Además, esos comportamientos humanos interfieren directamente en la gobernanza sobre estos bienes comunes, degradando también las relaciones sociales (HESS; OSTROM, 2017).

Es en este momento que la teoría de Elinor Ostrom se contrapone, como ya mencionado, a los modelos ortodoxos sobre el uso y la gestión de los recursos comunes, especialmente a la teoría de Garrett Hardin. Eso porque diferentemente de la Tragedia de los comunes, Ostrom $(2005,2010)$ creía - a partir de su extenso análisis empírico- que la gestión racional de esos recursos no dependía de la intervención directa del Estado o de la gestión por el propio mercado, estos por veces genéricos y mutilantes, sino por las propias partes interesadas en este bien común, mediante una acción colectiva fuerte basada en sólidos mecanismos de gobernanza construidos a través de la cooperación.

Del mismo modo, por creer que estos mecanismos o sistema de gobernanza sobre los recursos comunes no aceptaban modelos infalibles o listos, Ostrom (2005) objeta que a lo largo de sus estudios desde los años de 1980, se buscó identificar reglas específicas y comúnmente utilizadas por los más variados sistemas que sobrevivieron durante un largo periodo de tiempo, o sea, modelos de gobernanza que revelaran una gestión duradera de los bienes comunes mediante reglas operacionales planificadas y modificadas a lo largo del tiempo a partir de efectivas elecciones colectivas. Por otro lado, Ostrom (2005) no ha logrado identificar cualesquiera reglas consistentemente asociadas a un método ideal, hecho que la llevó a no buscar más por reglas específicas, sino por principios que caracterizarían las instituciones robustas de gestión del bien común.

Sin embargo, al revés de crear suposiciones meramente teóricas para tales principios, Ostrom las construye a partir del análisis empírico de esos preceptos en las más diversas iniciativas analisadas, verificando en la práctica que los sistemas robustos de gestión absorbieron la mayoría de esos principios, mientras que los sistemas que fracasaron o que no fueron capaces de gestionar sustentablemente los bienes comunes, tales principios no eran observados o debidamente estructurados (OSTROM, 2005).

Los principios derivados de esos estudios son (OSTROM, 2005, p. 259): 
1. Clearly defined boundaries. The boundaries of the resource system (eg, irrigation system or fishery) and the individuals or households with rights to harvest resource units are clearly defined.

2. Proportional equivalence between benefits and costs. Rules specifying the amount of resource products that a user is allocated are related to local and to rules requiring labour, materials, and/or money inputs.

3. Collective choice arrangements. Many of the affected by harvesting and protection rules are included in the group who can modify these rules.

4. Monitoring. Monitors, who actively audit biophysical conditions and user behaviour, partially accountable to the users and/or are the users themselves.

5. Graduated sanctions. Users who violate rules-in-use are likely to receive graduated sanctions (depending on the seriousness and context of the offense) from other users, from officials accountable to these users, or from both.

6. Conflict resolution mechanisms. Users and their officials have rapid access to low-cost, local arenas to resolve conflict among users or between users and officials

7. Minimal recognition of rights to organize. The rights of users to devise their own institutions are not challenged by external governmental authorities, and users have long-term tenure rights to the resource. For resources that are parts of larger systems:

8. Nestled enterprises. Appropriation, provision, monitoring, enforcement, conflict resolution, and governance activities are organized in multiple layers of nested enterprises (based on E. Ostrom 1990, 90).

Como se observa, cuando las actividades de definición, abastecimiento monitoreo, coacción constructiva, resolución de conflictos y gobernanza pueden ser organizados en múltiples camadas de empresas anidadas y también hay un buen conjunto de reglas en funcionamiento, existe un incentivo para la cooperación. Así pues, esa cooperación puede ser administrada por pequeños grupos de utilizadores de esos mismos recursos, sea en larga escala gradativamente acumulada de abajo hacia arriba, en distintos niveles. Esa percepción se origina de la propia interpretación de Ostrom (2005) sobre los principios, especialmente cuando la autora pondera que mientras los seis primeros principios buscan mejorar la sustentabilidad en el uso de recursos comunes por individuos locales, por pequeños grupos, los principios séptimo y octavo son direccionados a un nivel más alto de gobernanza, típicos de grandes grupos de individuos, instituciones y también naciones. 
Contodo, Ostrom (2005) pondera esas percepciones en su construcción argumentativa y señala que una manera de pensar sobre las implicaciones prácticas de los principios sería provocar cuestiones sobre los reales desafíos enfrentados en esta gestión del bien común. Una de las cuestiones sugeridas por la autora, tres reflexiones llaman la atención por acercarse - cuando analisadas conjuntamente- de las propias reflexiones que surgen de grandes estructuras de gobernanza del bien común, cuales sean, las propuestas de pensamiento sobre el tercero, séptimo y octavo principios.

Según la autora, para alcanzar el (tercer) principio de arreglos de elección colectiva, una alternativa sería pensar de qué forma los grupos pueden aumentar la participación de los involucrados en la toma de decisiones clave sobre el sistema, incluso en relación a la creación y modificación de reglas que se adapten a la realidad de esos grupos. En lo que se refiere a la efectividad del (séptimo) principio de reconocimiento mínimo de derechos de organización, el cual preconiza el derecho de que los individuos posean ese bien común a largo plazo y con sustentabilidad, podría alcanzarse a partir de esfuerzos funcionales y creativos autogestionados en la elaboración de mecanismos de administración eficaces de esos recursos. Finalmente, en los casos de grandes sistemas de gestión de recursos comunes, el (octavo) principio de empresas anidadas sería capaz de ser efectivo a través del desarrollo de un sistema policéntrico y dinámico de gobernanza, ejercidos de abajo hacia arriba y en múltiples camadas de decisiones (OSTROM, 2005).

Esa construcción de un modelo de gestión policéntrica basado en el reconocimiento de derechos a partir de la creación de arreglos colectivamente elaborados, replica Ostrom $(2005,2010)$, se fundamenta en dos verificaciones empíricas a lo largo de las décadas de estudio, las cuales se caracterizan como amenazas a la gestión del bien común, especialmente en relación a los fallos en la transmisión de la información y en el ejercicio de la acción colectiva.

La primera verificación está en el hecho de que cuando las desiciones no son plenamente discutidas entre los miembros, hay la tendencia de creación de liderazgos que se sostienen en coaliciones formadas a través de paternalismo, de la coerción y/o de la corrupción en detrimiento de la voluntad general de los individuos. Del mismo modo, al verse alejados de las propias reglas y del grupo central de decisiones, surge la segunda amenaza, en la cual los participantes ven las reglas del grupo como obstáculos a ser superados, y no la representación escrita de la voluntad colectiva. Como consecuencia de este escenario se tiene la presión por diversas formas de interpre- 
taciones de las reglas, llevándolas a un debilitamiento general (OSTROM, 1999; 2005; 2010).

Como se percibe, es en esta complejidad de los sistemas y de la acción humana - los cuales no permiten modelos acabados- que Ostrom y sus compañeros de la academia concentran sus esfuerzos para desarrollar no solo una comprensión más completa de esas recurrencias, sino también proponer una forma de gobernanza que alcance en esas múltiples camadas de decisión y garantice substancial conocimiento sobre el bien común a ser gestionado: la gobernanza policéntrica (OSTROM, 2010).

Con relación a la definición y capacidades de adaptación de un sistema de gobernanza policéntrica, la autora es didáctica al afirmar que (OSTROM, 2005, p. 283):

By polycentric I mean a system where citizens are able to organize just one but multiple governing authorities at differing scales (see V Ostrom, Tiebout, and Warren 1961; Ostrom 1997, 1999). Each unit exercises considerable independence to make and enforce rules within a circumscribed domain of authority for a specified geographical area. In a polycentric system, some units are general-purpose governments while others may be highly specialized. Self- organized resource governance systems in such a system may be special districts, private associations, or parts of a local government. (...)

In a polycentric system, the users of each common-pool resource would have some authority to make at least some of the rules related to how that particular resource will be utilized. Thus, they would achieve most of the advantages of utilizing local knowledge as well as the potential to learn from others who are also engaged in a similar trial-and-error learning process in parallel systems (Folke, Berkes, and Colding 1998). On the other hand, problems associated with local tyrannies and inappropriate discrimination can be addressed by larger, general-purpose governmental units who are responsible for protecting the rights of all citizens and for the oversight of appropriate exercises of authority within smaller units of government. (...) Because polycentric systems have overlapping units, information about what has worked well in one setting can be transmitted to others who may try it their settings. Associations of can be encouraged to speed up the exchange of information about relevant local conditions and about policy experiments that have proved particularly suc- 
cessful. And, when small systems fail, there are larger systems to call upon - and vice versa ${ }^{4}$

Lo que el concepto y su importancia revelan es que la gobernanza policéntrica es nada más que la ampliación del conocimiento de los recursos comunes -y consecuentemente de los procesos de toma de decisión originados de su gestión- al mayor número posible de sus legatarios, promoviendo la democratización de las discusiones de interés en todas las escalas de decisión. En otros términos, al promoverse un modelo de gobernanza que coleccione decisiones heterogéneas actuando de forma altiva, bajo un sistema común de reglas, Ostrom (1999; 2005; 2010) cree que tales decisiones que involucren el bien común serán realmente productiva y sustentable.

Esa premisa de decisiones heterogéneas e independientes vinculadas a una idea central surge de la profundización de Ostrom sobre los estudios acerca de la policentralidad iniciados por su esposo Vicent (HESS; OSTROM, 2007), el cual a partir de los estudios de Michael Polanyi ${ }^{5}$ so-

${ }^{4}$ Por policéntrica entiendo como un sistema en el cual los ciudadanos son capaces de organizar múltiples autoridades de gobernanza em diferentes escalas (ver $\vee$ Ostrom, Tiebout, e Warren 1961; Ostrom 1997, 1999). Cada unidad ejerce una independencia considerable para crear y aplicar las reglas dentro de un dominio circunscrito de autoridad para una área geográfica especificada. En un sistema policéntrico, algunas unidades son de propósito general, mientras que otras pueden ser altamente especializadas. Los sistemas de gobernanza de recursos auto organizados pueden ocurrir en distritos especiales, asociaciones privadas o formar parte de un gobierno local. En un sistema policéntrico, los usuarios de cada recurso común tendrían alguna autoridad para crear por lo menos algunas de las reglas relacionadas a la forma como ese recurso particular será utilizado. De esa forma, se lograría la mayoría de las ventajas en utilizar el conocimiento local, como también el potencial para aprender con otros que están involucrados en un proceso de aprendizaje de ensayo y error en semejantes sistemas (Folke, Berkes y Colding, 1998). Por otro lado, los problemas asociados a las tiranías locales y a la discriminación inadecuada se pueden abordar por unidades gubernamentales mayores y de propósito general, responsables por proteger los derechos de todos los ciudadanos y por la supervisión de ejercicios adecuados de autoridad en unidades de gobierno menores. Como los sistemas policéntricos poseen unidades sobrepuestas, las informaciones sobre lo que ha funcionado bien en una configuración pueden ser transmitidas para otras personas que pueden intentar sus configuraciones. Las asociaciones pueden ser incentivadas a acelerar el intercambio de informaciones sobre las condiciones locales relevantes y sobre las experiencias políticas que se revelaron particularmente exitosas. Cuando pequeños sistemas fallan, existen sistemas mayores para recorrer y viceversa.

${ }^{5}$ El desarrollo original de Michael Polanyi del concepto de policentrismo fue el resultado de su interés en las condiciones sociales preservando la libertad de expresión y el estado de derecho. El abordaje se basaba en su análisis social en una analogía con la organización de la comunidad científica, considerando que el éxito de la ciencia era resultado de un cierto tipo de organización social, y no de cientistas siguiendo un «método científico» rígidamente definido (POLANYI, 1951). 
bre la policentralidad en cuanto sistema social de muchos centros de decisión, con prerrogativas autónomas limitadas y operando bajo un conjunto que abarca diversas reglas, ha evolucionado su concepto en dos direcciones complementares: (i) creación de fundamentos de una teoría social construida alrededor del concepto de policentrismo; (ii) análisis empírico de esos casos, en la compañía de Elinor, concentrándose en una variedad de estudios bajo la perspectiva de un paradigma policéntrico (OSTROM; OSTROM, 2014).

De acuerdo con McGinnis (1999), la visión de Vicent y Elinor Ostrom sobre este paradigma policéntrico en ambientes de toma de decisiones amplios se originaba justamente de la contradicción a la ortodoxa visión dominante sobre el monopolio en cuanto elemento clave del ejercicio de poder. Aunque reconocieran que un sistema monocéntrico no impide la existencia de elementos de policentricidad en la gestión, y viceversa, la crítica al sistema monocéntrico está en el hecho de que este aplica reglas de poder en una única estructura de decisión que posee cierto monopolio. En ambientes colectivos ese monopolismo costumbra disminuir la legitimidad de las decisiones, que serán mejor adaptadas si diversas estructuras de decisión con ejes comunes sucedieran de decisiones relativamente autónomas para determinar, reforzar y alterar las relaciones legales, resultando así en una mayor participación de los actores involucrados.

Así pues, en un sistema político policéntrico no hay monopolio definitivo sobre el ejercicio del poder, y los representantes de esos individuos están restringidos a los límites ideológicos, legales y regimentales admitidos por este grupo. Esta ausencia de centralidad puede, en un primer momento, transparecer una sensación de caos en los procesos de toma de decisiones. Sin embargo, los conflictos originados de las discusiones acerca del bien común pueden generar la necesidad de más informaciones sobre ese recurso, promoviendo entre los participantes la gradual y constante busca de esfuerzos para gestionar y aclarar los problemas comunes a través de la cooperación (OSTROM, 2005).

Concluyendo, lo que la gobernanza policéntrica demuestra -igualándose al pensamiento cooperativista- es que el ejercicio del poder por los propios interesados, en sus diversas escalas, preserva el interés común cuando establecido a través de mecanismos de gobernanza propios, eficientes y cooperativos, evitando las tragedias de gran escala. Confirmar a los propios interesados la efectiva participación y la garantía de que sus decisiones serán, al menos, debatidas y decididas en conjunto, significa alejar de la gestión común el mero ejercicio de la gobernanza en entidades centrales, monocéntricas, distantes de la 
realidad local y sin conocimientos de las condiciones regionales. De esa forma se evita la degradación de los recursos comunes (OSTROM, 2010).

Sin embargo, la confirmación de la gobernanza policéntrica y de sus principios para la gestión de los bienes comunes (Common Pool Resources) de acuerdo con Ostrom (2005) pasa por la utilización de ese modelo y sus principios en la práctica, estresándolos en los más variados escenarios, pues, los sistemas policéntricos son, por si solo, complejos y adaptativos. Por otro lado, en los casos donde la cooperación es la premisa de actuación y donde las normas de gobernanza consideran como punto de partida la reciprocidad y la confianza en nombre de la sustentabilidad del bien común, la gestión policéntrica tendrá grandes oportunidades de prosperar, ya que «self-organizing arrengements enable people to learn more about on another's needs» (OSTROM, 2005, p. 288), o sea, tales arreglos autogestionados permitem que las personas aprendan más sobre los demás. Así que, esta es una premisa histórica de la democracia cooperativa.

\section{La Gestión Democrática como premisa de las sociedades cooperativas}

Los grandes cambios sociales y económicos que atraviesan el proceso de globalización siempre han traido, y todavía traen, la discusión de alternativas organizacionales que agreguen la población y entiendan la realidad social y sus vínculos a partir de sus aspectos políticos, económicos sociales y educativos, siempre considerando, contodo, la participación efectiva de las personas en la busca de alternativas económicamente viables.

Delante de esas exigencias actuales se percibe que las cooperativas siguen siendo, aunque después de 170 años desde la creación de la primera - los Pioneros de Rochdale - una de las alternativas y formas más avanzadas de organización de la sociedad, al final, además de ser técnicamente ejecutable y socialmente deseables, tal forma de organización socioeconómica de administración autogestionada ya ha traido respuestas históricas para la generación de empleos y la redistribución de renta en todo el mundo (ZEULI; CROPP, 2004).

Eso explica en la medida en que la aplicación de las ideas cooperativistas para la transformación de las relaciones de trabajo y la mejora de calidad de vida de la población siempre estuvo pautada en el estricto respeto a sus valores y principios. La perennidad del Cooperativismo está basado sobre un conjunto de ideas y nociones amplias y al- 
truistas como la mutualidad, la unión de esfuerzos, la solidariedad, la no exploración del hombre por el hombre, la justicia social, la democracia, la augestión. De esa forma, se puede afirmar que los casos de éxito del Cooperativismo siempre se justificaron en sus propios ideales y en la convicciones de sus miembros, dedicados en una acción común que garantizara su desarrollo en bases democráticas, y repudiando a cualquier paternalismo que relativizara la acción colectiva de sus miembros (SCHNEIDER, 1991).

Por eso, aunque en el Cooperativismo la acción social y la defensa de su pleno desarrollo clamen por una acción cooperativa que presupone la integración de todos sus valores y principios, la importancia de sus preceptos democráticos autoriza un recorte metodológico que busque identificar la defensa de la democracia como uno de sus compromisos históricos del Cooperativismo, al final, como destaca Namorado (2001), la organización cooperativa evoluciona al sabor de su proceso histórico, llevando de esa manera a incorporarse a un ideal de sociedad sin exploración.

De acuerdo con lo expuesto, el ejercicio de la cooperación desde los precursores del Cooperativismo moderno, ejercitado con el pionerismo conceptual por los 28 tejedores ingleses del condado de Lancachire, ha tenido como elemento central el deber de ultrapasar los muros del interés individual para la construcción colectiva capaz de transformar un entorno debilitado por las desigualdades de la revolución industrial (HOLYOAKE, 1975).

Eso significa decir que las reflexiones traidas por los Pioneros en sus estatutos constituyeron no solo una respuesta a las necesidades sentidas, como también a la diseminación de calidades por ellos apreciadas a partir de lo que les era singular frente a la realidad que los confrontaba; lo que se esperaba de sus miembros era una actitud cooperacionista basadas en estas reflexiones (MIRANDA, 2017).

El espíritu democrático es la espina dorsal del pensamiento rochaleano en la medida en que la participación de los miembros sobre bases democráticas es la condición del primer principio por la Rochdale Equitable Pioneers Society Limited, preconizando claramente que "voting is by members on a democratic (one-member, one-vote) basis» (ZEULI; CROPP, 2004, p. 9). Esa perspectiva, justifica Lambert (1975), se apoya en el empeño de los pioneros en la luchas del cartismo inglés por el sufragio universal, transfiriendo el principio político de la democracia a la cooperación.

Sin embargo, a pesar de tales razones políticas, lo que se tiene de concreto es que la clásica doctrina cooperativa -ampliamente corroborada por los autores de hoy- afirma que la democracia es el funda- 
mento crucial de la cooperación y que por veces con esta se confunde. Por tratarse de una sociedad de personas, las cooperativas representan a sus cooperados, operando con ellos y para ellos, cerrando un ciclo de interacción practicado por aquellos que nunca deben abdicar de su calidad en cuanto individuo (BULGARELLI, 1967; LAMBERT, 1975; CANABAL, 2009; MIRANDA, 2017).

Contodo, esta razón democrática de las sociedades cooperativas no está solamente en su perspectiva histórica y filosófica, pero se disemina ampliamente en la regulación legal de la organización. Por más que las decisiones colectivas sean ampliamente discutidas, se puede cuestionar y opinar acerca de cualquer decisión, motivo por el cual hay un momento donde el estado de discusión de temas no puede convertirse permanente en busca de un consenso. Aquí está la justificación del voto para la toma de decisiones. Por eso, dentro de las estructuras legales que conforman las sociedades cooperativas, la democracia no solo asegura la participación de las personas, sino también legitima la decisión tomada por la mayoría al final de un proceso de toma de decisiones (BULGARELLI, 2000; CANABAL, 2009).

Por lo tanto, se tiene que el principio cooperativo de la gestión democrática es nada más que la instrumentalización de la esencia democrática en las cooperativas, del valor cooperativo de la democracia, el cual este tercer enunciado señala que las sociedades cooperativas son organizaciones democráticas gestionadas por sus propios miembros, los cuales participan activamente en la determinación de sus políticas y toma de decisiones y eligen sus propios representantes ante el conjunto de los miembros (ACl, 2013).

Además, la $\mathrm{ACl}$ en su Declaración Sobre la Identidad Cooperativa corrobora dentro del principio de la gestión democrática que el ejercicio de esta garantía ocurre mediante derechos igualitarios de votación. Para tanto, Perius (2001) y Miranda (2017) claramente disponen que el órgano máximo de control del asociado son las asambleas generales, en las cuales se fundamenta la propia estructura democrática de las sociedades cooperativas bajo la premisa rochaleana de «un hombre, un voto».

En Brasil, esta premisa se encuentra en el artículo 4. ${ }^{\circ}$, inciso $V$, de la Ley Federal n. ${ }^{\circ} 5.764$ (BRASIL, 1971), que preconiza que las cooperativas son sociedades de personas que se distinguen de las demás sociedades, entre otros motivos, por la singularidad de los votos de sus miembros. Del mismo modo, el artículo 38 de la misma ley reconoce que la asamblea general es el órgano supremo de la sociedad con sus poderes para decidir — por el voto de cada miembro- los negocios relativos a su objeto, tomando aun las decisiones convenientes al de- 
sarrollo y defensa de la empresa común. De esa forma, la perenidad y la sustentabilidad de toda y cualquier cooperativa está fuertemente vinculada a la preservación de su perspectiva democrática, en la escucha sistemática y organizada de los miembros en la más amplia escala de participación posible.

No obstante, el ejercicio de la democracia en las cooperativas tiene sus costos y limitaciones, las cuales por veces llegan a ser utópico por el hecho ampliamente reconocido de que las asambleas generales no logran la participación directa de todos los socios. Hay también otro factor relevante al considerar que en el campo de las decisiones cooperativas prevalece el principio de la mayoría sobre el principio del veto, la insatisfacción de una minoría más actuante puede producir efectos exploratorios a costa de una mayoría asociada, que es apática y no participante (PERIUS, 2001).

Sumándose a esos retos, hay que reconocer todavía que aunque las cooperativas tengan históricamente raíces locales, no se debe ignorar la existencia cada vez mayor de grandes - o por veces pequeñas pero complejas - sociedades cooperativas que realizan sus actividades sin un centro físico definido, con miembros físicamente dispersos a tal punto que la convivencia y consecuente participación en los procesos de toma de decisiones se vuelve una tarea difícil y casi imposible.

De esa manera, es necesario comprender y emprender medidas que no solo fomenten la participación de los miembros, sino también garanticen que estos tengan voz además de sus estructuras administrativas físicas (HENRY, 2013).

Una de las alternativas compiladas por Cracogna Hagen y Fici (2013) a partir de las legislaciones de diversos paises del mundo fue la participación de los miembros en los procesos asambleares bajo la forma de delegación, o sea, mediante representación de prepuestos escogidos por los propios miembros en el goce de sus derechos sociales. Con relación a sus requisitos y procedimientos, Henry (2013, p. 90) es didáctico:

Pueden conformarse asambleas regionales y/o asambleas por sección cuando el tamaño de la cooperativa en términos de cobertura territorial o de cantidad de asociados fuera tal que el quórum necesario resulte difícil de alcanzar, o cuando los procedimientos de la asamblea general se convirtieran en demasiado engorrosos, o cuando lo requieran los diferentes intereses involucrados en el caso de una cooperativa multipropósito. Estas asambleas descentralizadas eligen a sus representantes ante una asamblea de delegados que reemplaza a la asamblea general. El orden del día de estas asambleas descentralizadas, así como sus modalidades de deliberación y votación, serán 
decididos a nivel central para garantizar la aplicación de las mismas normas en toda la cooperativa. Para reforzar la comunicación entre las diferentes partes, los integrantes del consejo de administración y de la comisión de vigilancia, si ésta existiera, deben participar en estas asambleas descentralizadas.

En el caso de Brasil (1971), el artículo 42 de la Ley Federal n. ${ }^{\circ} 5.764$ autoriza las sociedades cooperativas a adoptar el modelo de representación delegada a través de representantes elegidos por los propios asociados, desde que estos, en las cooperativas singulares, excedan a 3.000 (tres mil) asociados o residan a más de $50 \mathrm{~km}$ (cincuenta quilómetros) de la sede, estando a cargo del Estatuto Social, por su vez, establecer el número de delegados, la época y la forma de su elección por grupos seccionales de asociados de igual número y el tiempo de duración de la delegación. Además, el ortogamiento a los estatutos sociales cuanto a las reglas de delegación es también una premisa en otros paises.

Henry (2013), ratificado por Cracogna, Henry y Fici (2013), reconoce que cuando los asociados comprenden que ciertos requisitos son imprescindibles para la sociedad, la autogestión democrática de la cooperativa autoriza sus miembros a establecer sus reglas propias en sus estatutos y reglamentos, pues, lo que importa es el control democrático de los asociados. De esa forma, ponderan los autores, que los miembros tienen la posibilidad de crear sus propias reglas de representación con libertad, de acuerdo con la comprensión de su entorno.

Como consecuencia de esa libertad, estudiosos y cooperativas señalan las diferentes interpretaciones que se dan al modelo de representación delegada. Para Morais y Silva (2015), por ejemplo, la máxima rochaleana de "un hombre, un voto» debe ser incorporada a las disposiciones delegadas, proponiendo que a cada delegado sea disponible un voto en la asamblea general. Tal modelo es implementado en Brasil por las cooperativas de crédito que integran el Sistema Sicredi (2017), en las cuales los delegados, en los procesos asambleares, traen la decisión de su grupo establecida en las llamadas Asambleas de Núcleo, estas realizadas con los asociados presentes en cada pré asamblea. Por otro lado, modelos cooperativos como la Cajamar Caja Rural (2017), de España, preconiza que la representación delegada ocurre en las asambleas de forma directa, o sea, el delegado representa tan solamente los votos que les fueron confiados.

Aunque haya la posibilidad de elección entre cualesquiera de los modelos mencionados, los cuales deberán ser siempre previamente discutidos y dispuestos en sus estatutos y/o reglamentos específicos, lo 
que se percibe es una ausencia de justificativa teórica para la elección de determinados modelos, que debe representar, como visto, la consecución máxima del principio de la gestión democrática.

\section{La sociedad cooperativa en cuanto bien común y la policentricidad como fuente de defensa de la gestión democrática. Reflexión estimulada por Elinor Ostrom}

Una vez analisadas las dos bases teóricas de este estudio, o sea, la gobernanza policéntrica propuesta por Elinor Ostrom para la gestión democrática como premisa histórica, doctrinaria y legal de las sociedades cooperativas, lo que se pretente aquí es reflexionar como la policentralidad puede apoyar la construcción y el fortalecimiento de la acción común en el ejercicio de la democracia, especialmente en las grandes cooperativas que, como visto, tiene la oportunidad de delegar la representación.

En lo que se refiere a esa reflexión, se nota que al buscar en todos sus años de investigación conciliar la construcción de su teoría con una consistente base empírica en todos los rincones del planeta, Elinor Ostrom estimulaba la experimentación en los más diversos ambientes, justamente por creer que la complejidad de la acción humana siempre desafiará a los investigadores en busca de otras formas de organización que aumenten las oportunidades de adaptación y aprendizaje en un mundo de constantes e inciertos avances (OSTROM, 2005).

Tanto es así, que en una de las últimas coproducciones académicas antes de su desaparación, ocurrida en junio de 2012, Ostrom - juntamente con David Sloan Wilson y Michael E. Cox - ha instigado en el artículo Generalizing the core design principles for the efficacy of groups la generalización de la aplicabilidad de los principios de los bienes comunes bajo el fundamento de que cuando esos principios aumentan la eficacia de la gestión de esos bienes, los mismos podrían ser diseminados para otros tipos de grupos, permitiendo así su utilización como una estructura práctica destinada a aumentar la eficacia de varios tipos de grupos a partir de la dinámica evolución de la cooperación en nuestra propia especie. Y la justificación para eso ocurre por dos motivos centrales, según (WILSON; OSTROM; COX, 2013, p. S22):

First, they follow from the evolutionary dynamics of cooperation in all species and the evolutionary history of our own species. Second, because of their theoretical generality, we argue that the principles have a wider range of application than CPR groups and are relevant to nearly any situation where people must cooperate and coordinate 
to achieve shared goals. For both of these reasons, theprinciples can be used as a practical guide for increasing the efficacy of groups, although local tailoring is usually required for their implementation.

Como se percibe, al afirmar que los principios de los recursos comunes y de la gestión policéntrica poseen una generalidad teórica capaz de ampliar la gama de sus aplicaciones para casi todas las situaciones en que las personas deben cooperar y coordenar sus acciones para alcanzar objetivos compartidos, ocurre aquí un salutar acercamiento de la teoría de Elinor Ostrom con la doctrina cooperativa, expuesta por Lambert (1975) como un conjunto de doctrinas que confieren a la cooperación papel de importancia imprescindible para la solución de los problemas económicos y sociales de sus individuos.

Parece posible entonces que las nociones de policentralidad y de gestión policéntrica, por si solo, pueden justificar la construcción de un modelo de representación delegada que efectivamente transmita para las asambleas generales de grandes cooperativas, o incluso en los procesos de toma de decisiones de menor escala, la efectiva voluntad del miembro, fomentando su participación y defendiendo la gestión democrática en cuanto premisa del quehacer cooperativo. Como ya mencionado, tales premisas son objetivos del movimiento cooperativo en todo el mundo ( $\mathrm{ACl}, 2013)$.

Por otro lado, la policentralidad como fuente de defensa de la gestión democrática en las cooperativas gana todavía más importancia cuando verificado que estas, en su estructura conceptual dada por la $\mathrm{ACl}$ (2013), son definidas como una asociación autónoma de personas que satisfacen las aspiraciones y necesidades comunes mediante una empresa de propiedad conjunta y gestionada democráticamente. Transponiendo tales premisas para la la teoría de los recursos comunes, se nota que las sociedades cooperativas son, en la práctica, el bien común de sus miembros en la medida en que se trata de un recurso compartido por determinado grupo de personas (HESS; OSTROM, 2007).

En esta condición, el gradativo y constante fortalecimiento de la gestión democrática por las sociedades cooperativas encuentra respaldo en los análisis de Ostrom (2005), cuando esta refuerza que para la implementación de reglas de la utilización de los bienes comunes es imprescindible que las comunidades participen, de los involucrados directos - en el caso de las cooperativas, sus propios miembros- haciéndoles comprender que estos son parte de la solución y que sin ellas el problema no será resuelto. Así pues, esta es la premisa de la democracia cooperativa al presuponer la manifestación de la voluntad colectiva a través de la libre expresión de las opiniones, de la comprensión y 
del respeto a las divergencias, del amor a la argumentación y de la decisión colectiva (CHARTERINA, 1995).

Así pues, gestionar en la policentralidad significa resistir a las reglas impuestas de arriba hacia abajo, establecidas por líderes aislados o gestores elegidos que ignoran el espectro social que involucra los recursos comunes, la empresa cooperativa. Tales reglas, ponderan Hess y Ostrom (2007), provocan un alejamiento de los líderes y de las propias comunidades entre sí, colaborando para la tragedia presentada por Hardin. Ya para las cooperativas, este alejamiento es igualmente mutilado, pues desaparece sus preceptos identitarios al punto de desconfigurar justamente el actuar democrático, anulando la voluntad manifestada de las personas (premisa básica de la cooperación) para ceder a los intereses del capital. Cuando eso ocurre, la cooperativa perece, dejando de ser una cooperativa (MIRANDA, 2017).

En síntesis, es el clamor por la sustentabilidad de la cooperativa en cuanto recurso común a sus integrantes - basada en la cooperación y en la solidariedad - que fundamenta ese necesario y salutar acercamiento.

\section{Consideraciones y reflexiones finales}

En el objetivo de confirmar que la adopción de la representación delegada forma un sistema de gobernanza pluridemocrática que no afecta la esencia participativa de todos los miembros, y también protege la democracia en la gestión cooperativa defendida por la máxima de un hombre un voto, este ensayo teórico se propone a fundamentar - aunque de forma embrionaria - que la teoría de la gobernanza policéntrica desarrollada por Elinor Ostrom para la gestión de los recursos comunes es una importante herramienta teórica capaz de fortalecer la gestión democrática de las grandes sociedades cooperativas.

Por considerarse las legislaciones vigentes en el mundo que la representación delegada parte de la fragmentación del cuerpo social de grandes cooperativas en pequeños núcleos de deliberación y congraciamiento, se entiende que la gestión cooperativa podrá fortalecerse al acercarse de los principios de Ostrom. Eso porque los ocho principios - con especial atención al tercero, séptimo y octavo- se basan justamente en una gobernanza eficaz de los recursos, administrada por pequeños grupos del bien común que, con mayor facilidad, interactúan según un conjunto de reglas comunes. Eso es la propia esencia de la cooperación cooperativa.

Sin embargo, hay que reconocer que la confirmación de esa hipótesis deberá ser experimentada en estudios futuros, mediante observa- 
ciones de casos prácticos acerca de la gobernanza de la cooperativa en cuanto recurso común practicada en el seno de la representación delegada, incluso para el propósito de confirmar que el modelo de Ostrom preserva las raíces históricas democráticas del cooperativismo. Y para dar efecto práctico a eso es que el presente estudio, en un segundo momento, se propondrá a observar el ejercicio de la representación delegada en las cooperativas de crédito.

\section{Referencias}

Alejandro Martínez Charterina: «Los valores y los principios cooperativos», Revesco, v. 61, n. 1, nov. 1995, p.35-46. Disponível em: https://dialnet.unirioja.es/servlet/articulo?codigo=1148526>. Acesso em: 12 jul. 2017.

Böök, Sven Ake: Valores cooperativos para un mundo en cambio: informe para el Congreso de la ACl, ACl, Tokio, 1992.

BraSIL. Presidência da República: Lei n. ${ }^{\circ}$ 5.764, de 16 de dezembro de 1971. Define a Política Nacional de Cooperativismo, institui o regime jurídico das sociedades cooperativas, e dá outras providências. Disponible en <http:// www.planalto.gov.br/ccivil_03/LEIS/L5764.htm>. Acceso en: 17 jul. 2017.

Bulgarelli, Waldírio: Elaboração do Direito Cooperativo, Atlas, São Paulo, 1967.

- As sociedades cooperativas e a sua disciplina jurídica, Renovar, Rio de Janeiro, 2000, 2. ${ }^{\mathrm{a}}$. Ed.

Cajamar Caja Rural (España): Estatutos Sociales, Cajamar Caja Rural Sociedad Cooperativa de Crédito. 2017. Disponible en: <https://www.cajamar.es/ es/pdf/informacion-corporativa/estatutos-sociales.pdf>. Acceso en: 30 jul. 2017.

Canabal, Gerardo Montes: La Administración Cooperativa: Valores, Política y Poder en la gestión cooperativista, FCU, Montevideo, 2009.

Cracogna, Dante; HeNRY, Hagen; Ficl, Antônio (Ed.): International Handbook of Cooperative Law, Springer, Berlin, 2013.

ACI (Ed.): Plano de ação para uma década cooperativa, Bruselas, ACl, 2013.

HENRŸ, Hagen: Orientaciones para la legislación cooperativa, OIT, Ginebra, 2013, 2. ${ }^{\text {a }}$ ed.

Hess, Charlotte; Ostrom, Elinor: Understanding Knowledge as a Commons: from theory to practice, MIT Press, Cambridge, 2007.

HolyOAKE, George, J.: Historia de los pioneros de Rochdale, Intercoop, Buenos Aires, 1975.

ICA (Belgium): Exploring the co-operative economy, Report 2016, ICA, Brussels: 2016.

LAMBeRT, Paul: La Doctrina Cooperativa, Intercoop, Buenos Aires, 1975, 4. a. ed.

LAURIOLA, Vincenzo: Elinor Ostrom: «Um nobel heterodoxo e rosa-verde. Sinal de esperança?», Boletim da Sociedade Brasileira de Economia Ecológica, Brasília, v. 1, n. 21, ago. 2009, p.3-8,. Trimestral. 
McGInNIS, M.D.: Polycentricity and Local Public Economies: Readings from the Workshop in Political Theory and Policy Analysis, University of Michigan Press, 1999.

MIRANDA, José Eduardo de: De la crisis de identidad al rescate de la génesis del Cooperativismo, Dykinson S.L., Madrid, 2012.

- Filosofía Cooperativa, Juruá, Curitiba, 2017.

Morals, Roberto Tadeu Ramos; SILVA, Lázaro José da: «A participação nas decisões por meio da representação por delegados nas assembleias gerais do SICOOB COOPEMATER/PA», Revista de Gestão e Organizações Cooperativas - RGC, Santa Maria, p. 71-82. dez. 2015.

NAMORADO, Rui: Horizonte Cooperativo: política e projecto, Almedina, Coimbra, 2001.

Organização Das CoOperativas Do Brasil (Ed.): Agenda institucional do cooperativismo, Brasília, OCB, 2017.

Ostrom, Elinor: Understanding Institutional Diversity, Princeton University Press, Princeton, 2005.

- Governing the Commons: the evolution of institutions for collective action, Cambridge University Press, New York, 2010.

- «Beyond markets and states: polycentric governance of complex economic systems», Transnational Corporations Review 2.2, 2010, pp. 1-12.

- Design principles and threats to sustainable organizations that manage commons, Center for the Study of Institutions, Population, and Environmental Change, Workshop in Political Theory and Policy Analysis, Indiana University, 1999.

Ostrom, Vincent; Ostrom, Elinor: Choice, Rules and Collective Action, The Ostroms on the Study of Institutions and Governance, Ecpr Press, Colchester: 2014.

PerIUS, Virgílio Frederico: Cooperativismo e lei, Unisinos, São Leopoldo, 2001.

POLANYI, Michael: The Logic of Liberty, Routledge, New York, 1951.

SAlles, Letícia: «Biografia de Elinor Ostrom», Boletim da Sociedade Brasileira de Economia Ecológica, Brasília, v. 1, n. 21, ago. 2009, p.25-26. Trimestral.

SCHNEIDER, José Odelso: Democracia, participação e autonomia cooperativa, Unisinos, São Leopoldo, 1991.

SICREDI (Brasil): Assembleias: Voto por representação. 2017. Disponible en: $<$ https://pertencer.sicredi.com.br/assembleias>. Acceso en: 30 jul. 2017.

United Nation's Secretariat (Ed.): Measuring the Size and Scope of the Cooperative Economy: Results of the 2014 Global Census on Co-operatives, Dave Grace And Associates, Madison, 2014.

Williamson, Olivier: «Transaction-Cost Economics: The Governance of Contractual Relations». In: BuCKLEY, Peter \& MICHIE, Jhonathan (Ed): Firms Organizations and Contracts, Oxford University Press, 1996.

WILSON, David Sloan; OSTROM, Elinor; CoX, Michael E.: «Generalizing the core design principles for the efficacy of groups», Journal Of Economic Behavior \& Organization, [s.i.], v. 90, n. , jun. 2013, p.21-32,

Zeul, Kimberly A.; Cropp, Robert: Cooperatives: Principles and Pratices in the 21st Century, UW Extension, Madison, 2004. 